\title{
La pension libre complémentaire pour salariés (PLCS) en Belgique ${ }^{1}$
}

v. 19.12 .19

\section{Introduction}

1. Cette contribution retrace la genèse et explique le fonctionnement de la pension libre complémentaire pour salariés ou « PLCS ». Depuis le mois de mars 2019, la PLCS permet aux salariés du secteur privé de se constituer une pension complémentaire liée à leur activité professionnelle en l'absence de dispositions (suffisantes) à cet égard au sein de leur entreprise. Cette contribution fournit ensuite une analyse critique des caractéristiques de la PLCS en lien avec les mécanismes existant au sein des deuxième et troisième piliers de pension.

\section{Genèse et fonctionnement de la PLCS}

\subsection{De l'avant-projet à l'adoption de la Loi sur la PLCS}

2. En 2014, l'accord de Gouvernement prévoyait que « [1]es salariés auront la possibilité de se constituer volontairement une pension complémentaire dans le $2^{\mathrm{e}}$ pilier grâce à des retenues salariales effectuées par l'employeur. Les salariés déterminent librement le montant, dans certaines limites. Les avantages fiscaux seront les mêmes que ceux applicables aux régimes de pension complémentaire instaurés par les employeurs. ${ }^{2} \gg$ Le Gouvernement a déposé un projet de loi le 31 octobre $2018^{3}$ dans lequel il pose le principe de l'accès à la pension complémentaire pour l'ensemble de la population active. La nouvelle PLCS devait « apporter une solution pour les travailleurs qui ne se constituent toujours pas une pension complémentaire ou ne se constituent une pension complémentaire que d'un montant limité ${ }^{4}$. Pour le gouvernement, la PLCS s'inscrit dans la continuité des réformes visant à instaurer une pension complémentaire pour les autres catégories de travailleurs non-salariés ${ }^{5}$.

3. Le processus législatif débouche sur l'adoption de la loi du 6 décembre 2018 instaurant la PLCS ${ }^{6}$ et prévoit notamment l'adoption d'arrêtés royaux concernant certains paramètres d'application ${ }^{7}$ sur

\footnotetext{
${ }^{1}$ Pierre M. Sabbadini (pierre.sabbadini@uclouvain.be).

${ }^{2}$ Texte de l'accord de gouvernement du 9 octobre 2014 (« Un engagement économique, un projet social»), p. 41, disponible sur le site du Premier ministre de Belgique, https://www.premier.be/fr/accord-de-gouvernement, consulté le 11.11.19.

${ }^{3}$ Projet de loi instaurant une pension libre complémentaire pour les travailleurs salariés et portant des dispositions diverses en matière de pension complémentaire, résumé (p. 3), exposé des motifs (p. 4), avant-projet (p. 25), analyse d'impact (p. 48), avis du Conseil d'Etat (p. 52), projet de loi (p. 61), coordination des articles (p. 107), Doc. Ch. 2018-2019, n ${ }^{\circ} 3356 / 001,128$ p. Ce projet de loi a fait l'objet d'amendements (Doc. Ch. 2018-2019, n ${ }^{\circ}$ 3356/002, p. 6) et d'un rapport de la Commission des Affaires Sociales (Doc. Ch. 2018-2019, n 3356/003, p. 21) avant d'être adopté dans cette commission (Doc. Ch. 2018-2019, n $\mathrm{n}^{\circ} 3356 / 004$, p. 25) puis finalement en séance plénière (Doc. Ch. 2018-2019, n 3356/005, p. 24).

${ }^{4}$ Projet de loi instaurant une pension libre complémentaire pour les travailleurs salariés et portant des dispositions diverses en matière de pension complémentaire, résumé, Doc. Ch. 2018-2019, n 3356/001, p. 3.

${ }^{5}$ Voy. C. Devoet, La pension libre complémentaire des travailleurs salariés (PLCS), Actualités Fiscales, $\mathrm{n}^{\circ}$ 19/0101, 2019, p. 1.

${ }^{6}$ Loi du 6 décembre 2018 instaurant une pension libre complémentaire pour les travailleurs salariés et portant des dispositions diverses en matière de pensions complémentaires, M.B. 27.12.18, p. 103277, (ci-après la « Loi sur la PLCS ».)

${ }^{7}$ Article 3, paragraphe 3 et article 6 de la Loi sur la PLCS. En date du 18 décembre 2019, ces arrêtés royaux n'avaient pas encore été adoptés.
} 
lesquels la Commission des Pensions Complémentaires rendra un avis ${ }^{8}$. Le mécanisme de la PLCS entre en vigueur à partir de mars 2019.

\subsection{Fonctionnement de la Pension Libre Complémentaire pour Salariés (PLCS)}

\subsubsection{Définitions}

4. La Loi sur la PLCS fournit les définitions des concepts nécessaires à son application ${ }^{9}$ sauf celui de pension complémentaire libre pour salariés. En effet, le législateur choisit de passer par le concept de « pension complémentaire » qu'il définit comme : « la pension de retraite et/ou de survie en cas de décès de l'affilié avant ou après l'âge de retraite, ou la valeur en capital qui y correspond, qui sont constituées sur la base de versements de l'affilié conformément à une convention de pension en complément d'une pension fixée en vertu d'un régime légal de sécurité sociale ${ }^{10}$. Cette définition familière de la pension complémentaire diffère pourtant de celle qui figure dans la Loi sur les pensions complémentaires de 2003 dans la mesure où la « pension complémentaire » de 2003 repose sur des « versements obligatoires déterminés dans un règlement de pension ou une convention de pension $»{ }^{11}$

5. On peut déplorer ce procédé légistique consistant à définir le même terme de manière différente dans deux législations du même domaine juridique. Ceci est d'autant plus regrettable que ces deux concepts peuvent s'appliquer en même temps dans l'hypothèse où un travailleur aurait recours à la PLCS parce que le plan d'entreprise ne lui permettrait pas de cotiser jusqu'au plafond prévu par la Loi sur la PLCS. De plus, on peut relever que parmi les dispositions de la Loi sur la PLCS qui visent notamment à modifier l'article $145^{1}$ CIR92, le législateur ajoute l'hypothèse où «[...] les cotisations et primes personnelles visées à l'article $145^{1}, 1^{\circ}$, ont trait à une pension libre complémentaire pour les travailleurs salariés, telle que visée par le titre 2 de la loi du 6 décembre 2018 [...]». ${ }^{12}$ Ainsi, bien que la PLCS ne soit pas définie dans la loi de 2018, le législateur utilise ce concept lorsqu'il modifie d'autres lois.

6. Le lecteur peut néanmoins reconstruire le concept de PLCS sur la base des différents éléments fournis par le législateur. Le premier élément est celui de la catégorie du « travailleur » éligible à la constitution d'une PLCS que la Loi sur la PLCS définit comme « la personne occupée en exécution d'un contrat de travail $\gg .{ }^{13}$

7. Le deuxième élément se trouve dans le caractère résiduaire de la PLCS. En effet, un travailleur ne peut constituer une PLCS que dans deux hypothèses. La première vise le travailleur qui ne bénéficie pas d'une couverture prévue par l'employeur ou le secteur en matière de pension complémentaire. La seconde concerne celui dont la couverture existante ne permet pas de constituer des réserves suffisantes. Si ces deux hypothèses sont clairement décrites dans les travaux préparatoires ${ }^{14}$, elles ne ressortent pas clairement de la Loi sur la PLCS. En effet, cette loi prévoit une formule permettant de calculer le montant

\footnotetext{
${ }^{8}$ Article 21 de la Loi sur la PLCS. La Commission des Pensions Complémentaires est un organe consultatif composé de 23 membres représentant les intérêts des employeurs, des travailleurs, des organismes de pension actifs en Belgique, des pensionnés et incluant des experts. Elle a été instaurée par l'article 53 de la Loi relative aux pensions complémentaires et au régime fiscal de celles-ci et de certains avantages complémentaires en matière de sécurité sociale, M.B. 15.05.2003, p. 26407, (ci-après la « Loi sur les pensions complémentaires » ou « LPC »). Sa composition actuelle et ses avis sont disponibles à l'adresse suivante : $\underline{\text { https://perma.cc/T5ZW-CGRD. }}$

${ }^{9}$ Article 2 de la Loi sur la PLCS.

${ }^{10}$ Article $2,1^{\circ}$ de la Loi sur la PLCS.

${ }^{11}$ Article 3, paragraphe $1^{\text {er }}$ de la LPC.

12 Article 47 de la Loi sur la PLCS.

13 Article $2,2^{\circ}$ de la Loi sur la PLCS.

${ }^{14}$ Projet de loi instaurant une pension libre complémentaire pour les travailleurs salariés et portant des dispositions diverses en matière de pension complémentaire, amendements, Doc. Ch. 2018-2019, n 3356/001, p. 3.
} 
maximum qu'un salarié peut épargner au titre de la PLCS. ${ }^{15}$ Ce calcul lui permet de déterminer, le cas échéant, le caractère « insuffisant » de sa couverture. Cette condition sera remplie d'office si le salarié n'est pas couvert par un autre plan, sa contribution à déduire équivalant dans ce cas à 0 .

8. Le troisième élément est lié au cadre juridique dans lequel le travailleur peut exercer son droit à la constitution d'une PLCS en raison du caractère insuffisant ou absent d'autres contributions de pension complémentaire. Il s'agit de la conclusion d'une convention de pension définie par la loi comme « la convention en matière de pension complémentaire où sont fixés les droits et obligations de l'affilié, de ses ayants droit et de l'organisme de pension ainsi que les règles relatives à la constitution de la pension complémentaire et au paiement des prestations ». ${ }^{16}$ Le travailleur devient ainsi un «affilié », c'est-àdire un « travailleur qui a conclu une convention de pension » ou un « ancien travailleur qui continue à bénéficier de droits actuels ou différés conformément à la convention de pension $»{ }^{17}$

\subsubsection{Calcul des contributions au titre de la PLCS}

9. Le salaire de référence constitue le point de départ du calcul de la contribution annuelle maximale qu'un salarié peut verser à un organisme de pension. Ce salaire de référence ${ }^{18}$ correspond au montant brut du salaire utilisé pour le calcul du paiement des cotisations sociales que le travailleur a perçu deux ans avant l'année de contribution à la PLCS. Ce montant figure sur la fiche relative au compte individuel du travailleur $^{19}$. Pour 2019, le salaire de référence sera donc celui perçu en 2017.

10. Si le salarié ne bénéficie d'aucun autre plan de pension complémentaire, il peut contribuer auprès de son organisme de pension jusqu'à 1600 euros $^{20} \mathrm{ou}$, si son salaire de référence est supérieur à 53.333 euros, à un montant plus élevé correspondant à $3 \%^{21}$ de ce salaire de référence.

11. Si le travailleur salarié est déjà couvert par un ou plusieurs plan(s) de pension complémentaire au sein de son entreprise ou de son secteur, il peut contribuer à une PLCS si à travers ce(s) plan(s), ses contributions n'atteignent pas le montant de 1600 euros ou 3\% de son salaire de référence. Pour connaitre le montant auquel le salarié peut contribuer au titre de PLCS, il doit déduire un montant équivalent aux contributions (patronales et personnelles) ${ }^{22}$ effectuées à travers le(s) plan(s) existant ainsi qu'une partie du rendement du capital déjà accumulé à ce titre. ${ }^{23} \mathrm{Ce}$ montant est évalué sur la base de la

\footnotetext{
${ }^{15}$ Article 3, paragraphe 2, al. 2 de la Loi sur la PLCS. Le Roi peut modifier le pourcentage du salaire de référence en vertu de l'article 3, paragraphe 3 de la Loi sur la PLCS.

${ }^{16}$ Article 2, $4^{\circ}$ de la Loi sur la PLCS.

${ }^{17}$ Article $2,3^{\circ}$ de la Loi sur la PLCS.

${ }^{18}$ Article $2,7^{\circ}$ de la Loi sur la PLCS.

${ }^{19}$ Article $16, \S 3,1^{\circ}$, de l'arrêté royal du 8 août 1980 relatif à la tenue des documents sociaux, M.B. 27.08.1980, p. 9863.

${ }^{20}$ Le montant de 980 EUR est indexé à 1600 EUR sur la base du coefficient d'indexation visé à l'article 178, § 3 , alinéa 1er, CIR 92 avec une majoration de $2 \%$ pour prendre en compte le niveau d'inflation de 2018. (Voy. Projet de loi instaurant une pension libre complémentaire pour les travailleurs salariés et portant des dispositions diverses en matière de pension complémentaire, amendements, Doc. Ch. 2018-2019, n 3356/001, p. 8.) Ce montant est également utilisé en l'absence de salaire de référence. Il s'agit d'un montant maximum permettant uniquement au salarié de contribuer en deçà de ce montant.

${ }^{21}$ L'article 3, paragraphe 3 de la Loi sur la PLCS prévoit que ce montant peut être modifié par arrêté royal.

${ }^{22}$ B. Mariscal \& L. Niessen, Nouvel engagement de pension pour les travailleurs salariés : la PLCS, La Lettre Fiscale Belge, Févier 2019, n³28, p. 12 -18.

${ }^{23}$ La formulation technique figurant à l'article 3, paragraphe 2, al. 2 est la suivante : « La contribution annuelle maximale correspond, pour une année de constitution, à la différence, lorsqu'elle est positive, entre les montants a) et b), tels que déterminés comme suit : a) 3 p.c. du salaire de référence; A défaut de salaire de référence ou si le résultat du calcul des 3 p.c. du salaire de référence est inférieur à 980 euros, ce dernier montant est pris en considération. Ce montant minimum est indexé suivant les dispositions de l'article 178, $\S \S 1 \mathrm{er}$ et 3 , alinéa 1er, du Code des impôts sur les revenus 1992; b) la variation des réserves visées par la loi du 28 avril 2003, dont le montant correspond à la différence, lorsqu'elle est positive, entre : - le total de ces réserves, calculé au ler janvier de l'année qui précède l'année de constitution (n-1); - et le total de ces réserves, calculé au ler janvier de la deuxième année
} 
variation des réserves en pension complémentaire. Les travaux préparatoires avaient évoqué la difficulté liée au fait que les réserves constituées au $1^{\mathrm{er}}$ janvier d'une année ne sont connues qu'en fin d'année. ${ }^{24}$ Dès lors, le législateur a choisi de mesurer la variation des réserves durant la même période que celle utilisée pour le calcul du salaire de référence (ex. du 01.01.2017 au 01.01.2018). Cette partie du rendement du capital accumulé est calculée en appliquant une opération de capitalisation ${ }^{25}$ au montant des réserves. Le taux d'intérêt utilisé pour cette opération de capitalisation correspond à la moyenne sur les six dernières années (2012-2018) avant l'année de constitution (ex. 2019) du taux d'intérêt payé sur les obligations d'Etat ${ }^{26}$. Les informations concernant la variation des réserves permettant au travailleur de déterminer le montant maximal de ses cotisations devraient être disponibles via le portail Mypension.be. ${ }^{27}$

\subsubsection{Fournisseurs et produits}

12. Le travailleur salarié éligible à la constitution d'une PLCS doit choisir lui-même l'organisme avec lequel il va conclure une convention de pension ${ }^{28}$. Bien que la Loi sur la LPCS prévoie la possibilité pour l'employeur de conclure un accord-cadre avec un organisme de pension auprès duquel ses travailleurs peuvent ensuite conclure une convention de pension, ceux-ci restent libres quant au choix de leur organisme de pensions. ${ }^{29}$

13. La Loi sur la PLCS définit l'organisme de pension comme une entreprise d'assurance ${ }^{30}$ ou une institution de retraite professionnelle. ${ }^{31}$ En pratique, les salariés peuvent choisir de conclure une convention concernant leur PLCS auprès d'un assureur-vie (produit de branche 21 ou 23) ou d'un fonds de pension (institution de retraite professionnelle ou «IRP »). ${ }^{32}$

qui précède l'année de constitution (n-2), capitalisées au taux qui correspond au taux d'intérêt moyen des six dernières années calendrier précédant l'année qui précède l'année de constitution (n-1) des OLO sur dix ans. »

${ }^{24}$ Projet de loi instaurant une pension libre complémentaire pour les travailleurs salariés et portant des dispositions diverses en matière de pension complémentaire, avant-projet, Doc. Ch. 2018-2019, nº 3356/001, p. 12.

${ }^{25}$ L'opération de capitalisation d'un montant « $\mathrm{X}$ » au taux d'intérêt « $\mathrm{i}$ » se traduit par la formule suivante $(X \times(1+i))=$ montant capitalisé.

${ }^{26}$ Les « Obligations Linéaires/Lineaire Obligaties » ou « OLOs » sont « des titres d'emprunt public dématérialisés tenus dans un système de comptes auprès de la Banque nationale de Belgique et émis par tranches successives. Elles sont caractérisées par un taux d'intérêt nominal et une échéance identiques, constituant une ligne. Au sein d'une même ligne, les obligations linéaires présentent donc une substituabilité parfaite et sont dites fongibles. Elles sont émises sur le marché primaire et sont négociées jusqu'à leur échéance sur le marché secondaire qui constitue ainsi le marché de référence déterminant leur cours. » (Voy. Cour des Comptes, Les obligations linéaires, instruments de gestion de la dette publique, Bruxelles, avril 2001, p. 6, disponible à l'adresse suivante : https://perma.cc/6RB6-WFN6. Leur taux est notamment disponible sur le site de la Banque Nationale de Belgique (https://perma.cc/XS6H-H3SR).

${ }^{27}$ Projet de loi instaurant une pension libre complémentaire pour les travailleurs salariés et portant des dispositions diverses en matière de pension complémentaire, exposé des motifs, Doc. Ch. 2018-2019, n 3356/001, p. 12.

${ }^{28}$ Article 4 de la Loi sur la PLCS.

${ }^{29}$ Article 4, al. 2 de la Loi sur la PLCS.

${ }^{30}$ L'article $2,9^{\circ}$ de la Loi PLCS fait référence aux entreprises d'assurance belges et étrangères visées aux Livres II et III de la loi du 13 mars 2016 relative au statut et au contrôle des entreprises d'assurance ou de réassurance, M.B., 23.03.2016, p. 19856.

${ }^{31} \mathrm{~L}$ 'article $2,9^{\circ}$ de la Loi PLCS fait référence aux institutions de retraite professionnelle visées à l'article $2,1^{\circ}$ de la loi du 27 octobre 2006 relative au contrôle des institutions de retraite professionnelle, M.B., 10.11.2006, p. 60162.

${ }^{32}$ Voy. les informations pratiques fournies par la FSMA disponibles à l'adresse suivante : https://perma.cc/URP6YB7U. 
14. Dans le cadre d'une assurance-vie de la branche 21, le salarié bénéficie d'une protection du capital et d'un rendement annuel fixe garanti tandis que dans le cadre d'un produit de la branche $23^{33}$ ou dans un fonds de pension le capital placé est soumis aux fluctuations (positives et négatives) liées aux investissements sur les marchés financiers. ${ }^{34}$

15. En pratique, seules certaines entreprises d'assurance ont décidé de proposer un produit de PLCS. Certaines l'offrent soit en gestion libre sous la forme d'un produit de la branche 21 ou 23 ou d'une combinaison des deux, soit dans une formule de gestion du cycle de vie par l'assureur sur la base de choix de départ ${ }^{35}$. D'autres assureurs fournissent uniquement un produit de la branche 21 au titre de la PLCS $^{36}$.

\subsubsection{Règles de protection et d'information}

16. Le législateur a prévu que des règles permettant d'assurer une protection adéquate aux bénéficiaires et aux affiliés concernant les caractéristiques des produits qui peuvent rentrer dans le cadre de la PLCS pourront être fixées par arrêté royal. ${ }^{37} \mathrm{Au}$ travers de cette habilitation, la loi vise spécifiquement la possibilité de limiter le type de produits ou d'actifs dans lesquels la convention de pension permettra d'investir les contributions. ${ }^{38}$

17. D'autres mesures d'information, plus générales, sont imposées par le législateur à l'organisme de pension comme celle de produire un rapport annuel sur la gestion de la convention de pension. Ce rapport est communiqué sur simple demande aux affiliés. ${ }^{39} \mathrm{Il}$ contient notamment des informations relatives à la stratégie d'investissement à long et à court termes et la mesure dans laquelle les aspects sociaux, éthiques et environnementaux sont pris en compte dans ces investissements. L'organisme de pension y précise également le rendement des placements, la structure des frais et la participation aux bénéfices des affiliés (si celle-ci est prévue).

18. Cette stratégie d'investissement doit faire écho à la déclaration écrite sur les principles de la politique de placement que l'organisme de pension doit élaborer et maintenir à jour. ${ }^{40}$ Cette déclaration contient, au minimum, les méthodes d'évaluation des risques d'investissement, les techniques de gestion des risques mises en œuvre et la répartition stratégique des actifs eu égard à la nature et à la durée des obligations de pension complémentaire. ${ }^{41}$

19. Cette obligation d'information est renforcée lorsque, dans le cadre d'une convention de pension, l'affilié a souscrit à un produit pour lequel il supporte le risque de placement. Dans ce cas, il peut obtenir sur simple demande l'éventail des options éventuelles de placement et le portefeuille de placement existant, avec une description des risques et des coûts relatifs à ces placements. ${ }^{42}$ De manière générale, l'affilié dispose également du droit de demander à l'organisme de pension la déclaration relative aux principes

\footnotetext{
${ }^{33}$ Voy. L'Arrêté royal du 14 novembre 2003 relatif à l'activité d'assurance sur la vie, M.B. 14.11.2003, p. 55201 et l'Arrêté royal portant règlement générale relatif au contrôle des entreprises d'assurances, M.B. 11.04.1991, p. 7483.

${ }^{34}$ Voy. FSMA, supra.

${ }^{35}$ Voy. les informations sur le produit fourni par ces entreprises d'assurances, disponibles aux adresses suivantes : https://perma.cc/ZWE9-4ALD; https://perma.cc/S8TV-XNJ3.

${ }^{36}$ Voy. les informations sur un de ces produits disponibles à l'adresse suivante $:$ https://perma.cc/7SHE-CMT5.

${ }^{37}$ Article 6, al. 1 de la Loi sur la PLCS.

${ }^{38}$ Projet de loi instaurant une pension libre complémentaire pour les travailleurs salariés et portant des dispositions diverses en matière de pension complémentaire, exposé des motifs, Doc. Ch. 2018-2019, n 3356/001, p. 13.

${ }^{39}$ Article 14 de la Loi sur la PLCS.

${ }^{40}$ Article 13 de la Loi sur la PLCS.

${ }^{41}$ Article 13, al. 3 de la Loi sur la PLCS.

${ }^{42}$ Article 14, paragraphe 2, $3^{\circ}$ de la Loi sur la PLCS.
} 
de la politique de placement et les comptes et rapports annuels de l'organisme de pension. ${ }^{43}$ Le législateur permet à la FSMA d'adopter un règlement déterminant la forme et le contenu de ces informations.

\subsubsection{Changement d'organisme de pension et transfert des réserves acquises.}

20. La Loi sur la PLCS permet au travailleur de changer d'organisme de pension en mettant fin à la convention de pension pour en conclure une nouvelle. ${ }^{44}$ Cela implique que l'affilié a le droit de transférer les réserves acquises disponibles ${ }^{45}$ vers un autre organisme de pension qui gère les réserves conformément aux règles applicables à la PLCS. Le législateur prévoit à cet égard qu'aucune perte de participations bénéficiaires ne peut être mise à charge de l'affilié ni déduite des réserves acquises au moment du transfert. ${ }^{46}$ L'organisme de pension communique à l'affilié, par écrit ou par voie électronique et au plus tard dans les trente jours qui suivent la demande de transfert des réserves, le montant des réserves acquises. Le législateur indique également que le nouvel organisme de pension ne peut imputer des frais d'acquisition sur les réserves transférées. ${ }^{47}$

\subsubsection{Convention de pension}

21. Concernant la période précontractuelle, le législateur a prévu l'adoption d'un arrêté royal qui déterminerait les modalités et règles spécifiques d'information (ex. une mise en garde des affiliés dans le cadre de l'information précontractuelle) que l'organisme de pension potentiel devrait respecter lors de l'offre et de la conclusion d'une convention de pension. ${ }^{48}$

22. La convention contient les droits et obligations du salarié-affilié, de ses ayants droit et de l'organisme de pension ainsi que les modalités applicables aux phases d'accumulation des contributions et de paiement des prestations ${ }^{49}$. Parmi les mentions obligatoires, la convention doit indiquer l'âge de la retraite et ce, de manière cohérente, sans déroger à l'âge légal minimum de retraite. ${ }^{50}$

23. Une fois la convention conclue, l'organisme de pension la communique au nouvel affilié ${ }^{51}$ qui informe alors son employeur de sa volonté de constituer une PLCS. ${ }^{52}$ A cet effet, le travailleur salarié va lui communiquer les éléments essentiels de la convention afin de lui permettre d'exécuter la retenue sur salaire à transférer à l'organisme de pension au titre de contributions à la PLCS. Il s'agit notamment du montant et de la périodicité de la retenue, des données de contact et bancaires de l'organisme de pension ainsi que d'une attestation de l'existence de la convention de pension.

24. Le législateur octroie à l'employeur un délai de deux mois à partir de la réception de toutes les informations requises pour la mise en œuvre de la première retenue ou de sa modification ultérieure, à la demande du travailleur ${ }^{53}$. Dans l'hypothèse d'une cessation du contrat de travail, l'employeur mettra évidemment fin aux versements. La convention de pension restera cependant en vigueur. ${ }^{54}$

\footnotetext{
${ }^{43}$ Article 14, paragraphe 2 de la Loi sur la PLCS.

${ }^{44}$ Article 12, al. 1 de la Loi sur la PLCS.

${ }^{45}$ L'article 12, al. 3 de la Loi sur la PLCS prévoit en effet que le transfert ne peut porter que sur la partie des réserves qui n'a pas fait l'objet d'une avance ou d'une mise en gage ou qui n'a pas été affectée dans le cadre de la reconstitution d'un crédit hypothécaire.

${ }^{46}$ Article 12, al. 2 de la loi sur la PLCS.

${ }^{47}$ Article 12, al. 4 de la Loi sur la PLCS.

${ }^{48}$ Article 6 de la Loi sur la PLCS.

${ }^{49}$ Article 2, al. 4 de la Loi sur la PLCS.

${ }^{50}$ Article 5 de la Loi sur la PLCS.

${ }^{51}$ Article 5, paragraphe 2 de la Loi sur la PLCS.

${ }^{52}$ Article 7 de la Loi sur la PLCS.

${ }^{53}$ Article 7, paragraphes 1 et 3 de la Loi sur la PLCS.

${ }^{54}$ Article 11 de la Loi sur la PLCS.
} 
25. Le législateur précise que les contributions retenues par l'employeur ne pourront pas venir en déduction de la rémunération de l'affilié pour l'application des règles du Code Judiciaire relatives à la saisie sur salaire. ${ }^{55}$

26. L'organisme de pension doit communiquer annuellement à l'affilié une fiche de pension contenant les éléments d'information importants concernant les montants des réserves ${ }^{56}$ et des prestations ${ }^{57}$ acquises auxquelles il a droit sur la base de la convention de pension ${ }^{58}$. Cette fiche peut éventuellement être transmise de manière électronique et l'organisme de pension peut en déléguer la communication sur la base d'une convention avec l'asbl SIGeDIS, notamment en charge de la base de données DB2P sur les pensions complémentaires. ${ }^{59}$

27. La loi prévoit également des échanges d'information entre l'organisme de pension et SIGeDIS pour la mise à jour de la base de données DB2P durant la période d' accumulation ${ }^{60}$ et lors de la mise à la retraite du travailleur ${ }^{61}$.

\subsubsection{Retenues sociales et régime fiscal applicable à la PLCS}

28. En ce qui concerne les contributions, le traitement fiscal de la $\operatorname{PLCS}^{62}$ comprend l'application de la taxe sur les opérations d'assurance ${ }^{63}$ et leur inclusion au sein de la catégorie éligible à une réduction d'impôt de $30 \%$ pour l'épargne à long-terme ${ }^{64}$.Une réduction de $30 \%$ sur le précompte professionnel dû sur le salaire est également appliquée sur les contributions retenues par l'employeur ${ }^{65}$, dans les limites de la règle de $80 \%{ }^{66}$.

\footnotetext{
${ }_{55}^{55}$ 'article 8 de la Loi sur la PLCS fait référence à la Cinquième Partie V, Titre $1{ }^{\text {er }}$, Chapitre V du Code judiciaire. ${ }^{56}$ Les réserves acquises auxquelles l'affilié a droit en vertu de l'article 9 de la Loi sur la PLCS sont définies à l'article $2,5^{\circ}$ de la Loi sur la PLCS comme « les réserves auxquelles l'affilié a droit, à un moment déterminé, conformément à la convention de pension».

${ }^{57}$ Les prestations acquises auxquelles l'affilié a droit en vertu de l'article 9 de la Loi sur la PLCS sont définies à l'article $2,6^{\circ}$ comme « les prestations auxquelles l'affilié peut prétendre à l'âge de retraite, conformément à la convention de pension, s'il laisse ses réserves acquises auprès de l'organisme de pension sans versement ultérieur de contributions».

${ }^{58} \mathrm{La}$ Loi sur la PLCS prévoit aux articles 9 et 10 une liste détaillée d'informations qui doivent figurer dans la fiche de pension et laisse au Roi et à la FSMA la possibilité d'en préciser le contenu et le format.

${ }^{59}$ Article 10, paragraphe 5 de la Loi sur la PLCS. La base de donnée DB2P sur les pensions complémentaires créée par les Articles 305 et 306 de la Loi-Programme (I) du 27 décembre 2006, M.B 28.12.2006, p. 75178, est consultable à l'adresse suivante : https://www.db2p.be.

${ }^{60}$ Article 10, paragraphe 6 de la Loi sur la PLCS.

${ }^{61}$ Article 11 al 3 de la Loi sur la PLCS.

${ }^{62}$ Voy. Partie 7 - La cessation de l'activité professionnelle et les pensions (p. 537 et suiv.) in O. D'Aout, D. Darte, N. Honhon e.a. L'impôt des personnes physiques, Coll. Les manuels pratiques, Anthemis, Limal, 2019, p. 698. Voy. C. Devoet, op. cit. 2019, p. 1.

${ }^{63}$ Article $175^{1}$, paragraphe 2 du Code des droits et taxes divers, tel que modifié par l'article 51 de la Loi sur la PLCS.

${ }^{64}$ Article $145^{1}, 1^{\circ}$ bis du CIR92 tel que modifié par les articles 45 à 50 de la Loi sur la PLCS. Voy. B. Mariscal et L. Niessen, op. cit., p. 5.

${ }^{65}$ Voy. L'article 88 CIR 92 et l'annexe III à l'AR/CIR 92, chapitre II Rémunération des travailleurs et revenus y assimilés, section 1, c) de l'AR/CIR 92 tel que modifié par l'Arrêté royal du 7 décembre 2018 modifiant, en matière de précompte professionnel, l'AR/CIR 92, M.B. 13 décembre 2018, p. 98477.

${ }^{66}$ Cette règle prévue à l'article 59 CIR92 soumet la déductibilité à titre de frais professionnels de certaines cotisations et primes patronales notamment à la condition que les prestations légales et extra-légales en cas de retraites (exprimées en rentes annuelles) ne dépassent pas $80 \%$ de la dernière rémunération brute annuelle normale et tiennent compte d'une durée normale d'activité professionnelle. Voy. B. Mariscal et L. Niessen, op. cit., p. 17. Voy. Projet de loi instaurant une pension libre complémentaire pour les travailleurs salariés et portant des dispositions diverses en matière de pension complémentaire, exposé des motifs, Doc. Ch. 2018-2019, n 3356/001, p. 23.
} 
29. En ce qui concerne les prestations, elles sont considérées comme des revenus de pension soumis à l'imposition ${ }^{67}$ à un taux de $10 \%{ }^{68}$ lorsqu'ils ont lieu à l'occasion de la mise à la retraite, du décès, ou à partir de l'âge de 60 ans (sauf si le cumul des revenus est plus avantageux pour l'affilié) ${ }^{69}$. Dans les autres cas, les prestations seront imposées au taux de $33 \%{ }^{70}$.

30. De plus, les prestations sont soumises à la cotisation INAMI de 3,55\% et à la cotisation de solidarité progressive de 0 à $2 \%$. Cette cotisation s'applique aussi en cas de décès sauf lorsque les prestations sont payées en cas de décès à un bénéficiaire autre que le conjoint survivant. ${ }^{71}$

\subsubsection{Contrôle}

31. Le législateur a confié le contrôle du respect des dispositions de la Loi sur la PLCS à la FSMA ${ }^{72}$. Il prévoit ainsi que la FSMA doit recevoir une communication de la liste des conventions de pensions conclues par les organismes de pension selon les modalités qu'elle fixe concernant la périodicité, le contenu et le support ${ }^{73}$. La FSMA peut demander toute information aux organismes de pensions afin de s'assurer du respect de la Loi sur la PLCS et de ses arrêtés ${ }^{74}$. Elle dispose également de pouvoirs d'enquête sur site auprès des sièges des organismes de pension situés en Belgique ${ }^{75}$. En cas de constat de manquements, la FSMA peut fixer un délai permettant à l'organisme de pension d'y remédier ${ }^{76}$. Passé ce délai, elle peut adopter des injonctions, éventuellement assorties d'une astreinte ${ }^{77}$, à l'encontre des organismes de pension et les communiquer aux affiliés, aux bénéficiaires des conventions de pension et au public notamment par voir de presse ${ }^{78}$.

32. La FSMA peut également infliger une amende administrative d'un montant maximum de 2500000 euros pour le même (ensemble de) fait(s) ${ }^{79}$. La Loi sur la PLCS prévoit une obligation spécifique pour certains professionnels tels que les commissaires, actuaires et responsables de la fonction actuarielle, d'informer la FSMA de tout fait ou décision dont ils ont eu connaissance dans le cadre de leur mission et qui constitue une infraction à la Loi sur la PLCS et/ou à ses arrêtés d'exécution. Dans ce cadre, ces professionnels bénéficient d'une protection et d'une exonération de responsabilité quant au contenu des informations divulguées. ${ }^{80}$

\footnotetext{
${ }^{67}$ Article 34, paragraphe $\left.1,2^{\circ}, \mathrm{c} / 1\right)$ CIR92.

${ }^{68}$ Article $171,2^{\circ}$, c) CIR92.

${ }^{69}$ Article $171,2^{\circ}, \mathrm{b}$, tiret 1 CIR92

${ }^{70}$ Article $171,1^{\circ}$, d) CIR92. Voy. Projet de loi instaurant une pension libre complémentaire pour les travailleurs salariés et portant des dispositions diverses en matière de pension complémentaire, avant-projet, Doc. Ch. 20182019, n 3356/001, p. 23. Voy. B. Mariscal et L. Niessen, op. cit., p. 17 sur le traitement des avances sur prestations dédiées au financement de biens immobiliers ou au rôle de garantie d'un emprunt hypothécaire d'une PLCS.

71 Circulaire 2019/C/67 relative à la pension libre complémentaire pour les travailleurs salariés (PLCS), 19.07.2019, disponible sur www.fisconetplus.be. Voy. B. Mariscal, L. Niessen, op. cit., p. 16.

${ }^{72}$ Articles 15 à 20 de la Loi sur la PLCS.

${ }^{73}$ Article 16 de la Loi sur la PLCS. Les organismes de pension disposent également de la possibilité d'effectuer cette communication par le truchement de l'asbl SIGeDIS.

${ }^{74}$ Article 17, al. 1 et 3 de la Loi sur la PLCS.

${ }^{75}$ Article 17, al. 2 de la Loi sur la PLCS. L'inspection sur site du siège situé en Belgique d'un organisme de pension étranger doit être précédée d'une communication aux autorités compétentes de l'Etat membre d'origine.

${ }^{76}$ Article 18 de la Loi sur la PLCS.

${ }^{77}$ L'article 18, paragraphe 2 de la Loi sur la PLCS prévoit qu'à l'issue du délai pour la mise en conformité fixé par la FSMA et après que l'institution ou la personne a pu faire valoir ses moyens, la FSMA peut infliger une astreinte ne dépassant pas 50000 euros par jour calendrier de retard, ni 2500000 euros pour la méconnaissance d'une même injonction.

${ }^{78}$ Article 18 paragraphe 1 de la Loi sur la PLCS.

${ }^{79}$ Article 18 paragraphe 3 de la Loi sur la PLCS.

${ }^{80}$ Article 20, al. 2 de la Loi sur la PLCS.
} 
33. La Loi sur la PLCS a prévu des sanctions pénales pour les manquements constatés aux devoirs d'information vis-à-vis de la FSMA de la part des professionnels impliqués dans la fourniture de la PLCS $^{81}$. La FSMA devra également désormais inclure la PLCS dans le cadre de son rapport bisannuel sur les pensions complémentaires. ${ }^{82}$

\section{Analyse critique de la PLCS}

34. Partant du constat des défis auxquels le système belge de pension devait faire face, le Gouvernement a avancé que la solution passerait par l'expansion du deuxième pilier, qui constitue un complément à la pension légale, ayant pour objectif le maintien pour les pensionnés d'un niveau de vie similaire à celui de leur vie active ${ }^{83}$.

35. Deux solutions semblaient se présenter au législateur : soit imposer à tous les secteurs la mise en place d'une pension complémentaire minimum, soit offrir au salarié le droit d'initiative de constitution d'une pension complémentaire réservé jusqu'ici à l'employeur ou au secteur. Le Gouvernement a écarté la première option pour des raisons liées au contexte économique et pour ne pas «court-circuiter la concertation sociale qui est à l'origine du développement des pensions complémentaires au niveau sectoriel $»{ }^{84}$ Cette seconde raison était placée dans le contexte de la négociation d'un accord interprofessionnel (AIP) pour la période 2019-2020 qui devait aussi aborder la question de l'harmonisation des pensions complémentaires pour les ouvriers et les employés. ${ }^{85}$ On constate cependant que l'AIP 2019-2020 conclu et rendu obligatoire par arrêté royal n'a pas traité cette question. ${ }^{86}$

36. C'est donc la seconde option que le Gouvernement a choisie pour étendre le deuxième pilier. Il est parti du constat qu'un salarié ne pouvait se constituer une pension complémentaire au sens de la LPC que dans la mesure où son employeur ou le secteur dans lequel son entreprise était active avaient décidé de mettre en place un plan de pension. Le Gouvernement a cependant relevé que, bien que l'initiative de constituer une pension complémentaire (collective) n'était pas accessible au salarié, le concept d'une pension complémentaire individuelle pour salarié existait déjà en droit belge. Il s'agissait du droit de continuation du plan de pension applicable à tous les salariés d'une entreprise auquel le travailleur était affilié depuis au moins 42 mois et qu'il pouvait exercer à titre individuel auprès d'un nouvel employeur avec lequel un contrat de travail était conclu. ${ }^{87}$ Cependant, les conditions liées à l'absence de plan auprès du nouvel employeur et à la durée d'affiliation n'avaient pas rendu cette option populaire. Ainsi, au $1^{\text {er }}$ janvier 2017, moins de 3\% des salariés affiliés au moins à un plan de pension avaient fait usage de cette possibilité. ${ }^{88}$

\footnotetext{
${ }^{81}$ L'article 22 de la Loi sur la PLCS vise les administrateurs, gérants ou mandataires d'organismes de pension et les employeurs ou leurs mandataires.

${ }^{82}$ Article 19 de la Loi sur la PLCS.

${ }^{83}$ Voy. Projet de loi instaurant une pension libre complémentaire pour les travailleurs salariés et portant des dispositions diverses en matière de pension complémentaire, exposé des motifs, Doc. Ch. 2018-2019, n 3356/001, p. 4.

${ }^{84}$ Voy. Projet de loi instaurant une pension libre complémentaire pour les travailleurs salariés et portant des dispositions diverses en matière de pension complémentaire, Rapport de la Commission des Affaires Sociales, Doc. Ch. 2018-2019, n 3356/003, p. 9

${ }^{85} \mathrm{Ibid}$.

${ }^{86}$ Voy. le résumé de l'AIP sur le site de la Première Ministre : https://perma.cc/LUZ3-35SW

${ }^{87}$ Article 33 de la Loi sur les pensions complémentaires.

${ }^{88}$ Source : Chiffres concernant les pensions complémentaires au $1^{\text {er }}$ janvier 2017 issus de la base de donnée sur des pensions complémentaires (DB2P) à la date du $1^{\text {er }}$ mars 2018, disponibles à l'adresse suivante: https://perma.cc/Q4JK-W68M.
} 
37. Dès lors, le Gouvernement a créé une nouvelle formule pour le salarié afin qu'il dispose également du droit de se constituer individuellement une pension du deuxième pilier. La loi sur la PLCS a également mis fin, pour l'avenir, à la possibilité de continuation individuelle ${ }^{89}$.

38. Les partis de l'opposition ont formulé de nombreuses critiques lors des discussions à la Chambre à l'égard du choix du Gouvernement de créer une $\operatorname{PLCS}^{90}$. Le Conseil Académique des Pensions a également émis plusieurs réserves concernant ce concept ${ }^{91}$. Plus tard dans le processus de formalisation du concept de PLCS, le Conseil Académique des Pensions ${ }^{92}$ a adopté une position assez critique sur le texte de l'avant-projet du 29 juin $2018 .{ }^{93}$

39. En ce qui concerne les partenaires sociaux, pour la Fédération des employeurs de Belgique, la PLCS s'insère dans la préoccupation plus large des employeurs concernant le deuxième pilier, et notamment, l'obligation de garantie de rendement. La FEB a également insisté sur l'importance de placer l'ajustement des paramètres de la PLCS (ex. le niveau des contributions) au sein de la politique salariale dans le cadre de la concertation sociale. ${ }^{94}$ Elle a estimé par la suite que le caractère volontaire de l'initiative était positif dans la mesure où il ne crée pas de nouvelles obligations ou garanties pour les employeurs ${ }^{95}$. Pour les syndicats, la PLCS ne constitue pas un renforcement ou une démocratisation du deuxième pilier mais ressemble davantage à un produit du troisième pilier ${ }^{96}$ bien qu'il soit en fait moins intéressant que les possibilités offertes au sein du troisième pilier. ${ }^{97}$ Certains auteurs de doctrine ont également formulé quelques interrogations quant au succès de la formule PLCS. ${ }^{98}$

40. Les critiques et interrogations principales visaient le fait que la PLCS décourageait l'extension du deuxième pilier (au sens collectif) et qu'elle renforçait une logique d'individualisation, faisant peser l'ensemble des responsabilités (initiative, choix de l'organisme de pension, choix du produit, calcul de la cotisation maximale, collecte d'informations, etc.) sur le salarié. De plus, les obligations de l'employeur sont limitées à une retenue de salaire et ne comportent donc aucune garantie. Enfin la PLCS

\footnotetext{
${ }^{89}$ L'article 30 de la Loi sur la PLCS modifie l'article 33 de la Loi sur les pensions complémentaires de sorte qu'il ne s'applique qu'aux travailleurs ayant fait usage de cette faculté de continuation à titre individuel avant l'entrée en vigueur de la Loi sur la LPCS.

${ }^{90}$ Voy. Projet de loi instaurant une pension libre complémentaire pour les travailleurs salariés et portant des dispositions diverses en matière de pension complémentaire, Rapport de la Commission des Affaires Sociales, Doc. Ch. 2018-2019, n 3356/003, p. 9

${ }^{91}$ Note de politique générale (pensions), Chambre des Représentants, DOC54K1428/009, 30 octobre 2015, p. 16 : «Par ailleurs, la possibilité sera également créé pour les salariés de se constituer librement une pension complémentaire du deuxième pilier. Celle-ci sera financée au moyen de retenues salariales effectuées par l'employeur. Les salariés détermineront librement le montant de ces retenues dans certaines limites. Les avantages fiscaux seront les mêmes que ceux applicables aux cotisations personnelles versées dans des pensions complémentaires instaurées par les employeurs. »

${ }^{92}$ Le Conseil Académique des Pensions (ci-après le «CAP ») a été créé en 2015 par les articles 2 et 15 à 20 la Loi du 21 mai 2015 portant création d'un Comité national des Pensions, d'un Centre d'Expertise et d'un Conseil académique, M.B. 22.05.2015, p. 29654. Le Conseil académique est le successeur de la « Commission de réforme des pensions 2020-2040 » qui avait été créée en 2013 par les ministres des Pension et des Indépendants afin de formuler des propositions de réforme du système belge de pension. Les informations relatives à la composition, au fonctionnement et aux avis du CAP sont disponibles à l'adresse suivante $:$ https://perma.cc/85HA-3CZA.

${ }^{93}$ Voy. Conseil Académique des Pensions, Position sur l'avant-projet PLCS, 3 juillet 2018, p. 2, disponible à l'adresse suivante : https://perma.cc/B8XC-ZV4R.

${ }^{94}$ Voy. l'interview de Pieter Timmermans, AG Employee Benefits Magazine, ${ }^{\circ}{ }^{18}$, Septembre 2015, pp. 21-22, disponible à l'adresse suivante : https://perma.cc/767R-NRSS.

${ }^{95}$ Voy. Fédération des Entreprises de Belgique, Circulaire S.2019-005, La nouvelle pension libre complémentaire pour salariés (PLCS) - Loi du 6 décembre 2018, 25 janvier 2019, pp. 1, 2 et 4, disponible à l'adresse suivante : https://perma.cc/3QV2-D4CN.

${ }^{96}$ FGTB, Magazine « Syndicats », $n^{\circ}$ 20, 2018, p. 7, disponible à l'adresse suivante : https://perma.cc/3FL5-BR69.

${ }^{97}$ CGSLB, Magazine «Librement », février 2019, p. 6, disponible à l'adresse suivante $:$ https://perma.cc/YF7SUC9G; Voy. https://perma.cc/KXY6-C9TB, p. 7.

${ }^{98}$ C. Devoet, op. cit.; B. Mariscal \& L. Niessen, op. cit.
} 
favoriserait les salariés disposant d'un revenu plus important et leur permettant de dégager des moyens pour constituer une PLCS.

\subsection{Impact de la PLCS sur le deuxième pilier}

41. On peut considérer que l'architecture du système de pension belge repose sur un premier pilier comprenant les régimes de pension légale, un deuxième pilier regroupant les régimes de pension complémentaires liés à l'activité professionnelle et un troisième pilier d'épargne individuelle volontaire constituée en dehors du cadre professionnel ${ }^{99}$. Il ressort clairement de l'exposé général des travaux préparatoires que le gouvernement considère que la PLCS fait partie du deuxième pilier et tend à sa « généralisation » ou « expansion » ${ }^{100}$.

42. La doctrine considère que la PLCS adhère à certains principes de la $\mathrm{LPC}^{101}$. Premièrement, la liquidation des prestations s'effectue lors de la mise à la retraite ${ }^{102}$. Deuxièmement, l'affilié dispose du droit de transférer ses réserves vers un autre organisme de pension ${ }^{103}$. Troisièmement, le salarié peut utiliser le capital constitué via une mise en gage pour une avance sur prestation pour contribuer à son patrimoine immobilier situé au sein de l'EEE. ${ }^{104}$ Quatrièmement, les règles relatives à la transmission des informations vers la base de donnée DB2P et l'établissement des fiches de pension sont similaires ${ }^{105}$. Cinquièmement, les règles relatives à la prescription et à la compétences des juridictions du travail pour les litiges afférents aux droit et obligations des parties dans le cadre de la LPC et de la LPCS $^{106}$ sont similaires. Sixièmement, la LPCS abroge la possibilité prévue à l'article 33 de la LPC ${ }^{107}$, pour un travailleur salarié, de continuer un engagement de pension à titre individuel.

43. Cependant la PLCS est parfois considérée comme un produit hybride ou un « deuxième pilier bis $»^{108}$. Elle se rattache au deuxième pilier dans la mesure où elle est liée à l'activité professionnelle et que l'employeur effectue des retenues sur le salaire de l'affilié. Elle s'apparente également au troisième pilier dans la mesure où tant l'initiative de constitution que les choix liés au fournisseur, au produit, au montant, etc. dépendent uniquement du salarié.

\subsection{Inégalités au sein du deuxième pilier}

44. Le fait que la PLCS favoriserait les salariés disposant d'un revenu plus important et leur permettant de dégager des moyens pour constituer une PLCS a été critiqué. Il ne s'agit pas d'une critique limitée à la PLCS mais plutôt d'un trait commun aux produits du deuxième pilier. En effet, si le taux de couverture concernant les plans du deuxième pilier est passé de $40 \%$ en $2004^{109}$ à $75 \%$ de la population active en $2018,{ }^{110}$ ce taux de couverture cache des différences importantes au niveau de l'impact en terme de

\footnotetext{
${ }^{99}$ O. Bertrand et S. Scaillet, Fonctionnement actuel du système de pension belge, pp. 7 - 69 in B. Bayenet, M. Fontaine, D. Léonard et al., La réforme des pensions, pour quels enjeux sociétaux, Larcier, 2019, pp. 394.

${ }^{100}$ Projet de loi instaurant une pension libre complémentaire pour les travailleurs salariés et portant des dispositions diverses en matière de pension complémentaire, résumé, Doc. Ch. 2018-2019, n 3356/001, p. 1.

${ }^{101}$ B. Mariscal \& L. Niessen, op. cit., Section 3 - PLCE, produit du deuxième pilier.

${ }^{102}$ Article 11, paragraphe 1 de la LPCS et article 27 paragraphe 1 de la LPC.

${ }^{103}$ Article 12 de la LPCS, article 32, paragraphe 1 de la LPC.

${ }^{104}$ Article 11, paragraphe 1 de la LPCS, article 27 paragraphe 2 de la LPC.

105 Article 10 de la LPCS et article 26 de la LPC.

106 Article 23 de la Loi sur la PLCS et article 55 de la LPC. Voy. F. Burniaux, « Droit du travail - Pension libre complémentaire pour les travailleurs salariés », Bulletin de l’OECCBB, 2019, vol. 2019, n² 2, pp. 13-14.

107 Article 30 de la Loi sur la PLCS.

${ }^{108}$ Voy. Conseil Académique des Pensions, 3 juillet 2018, op. cit., p. 1.

109 Assuralia, Les plans sectoriels - guide à l'usage des partenaires sociaux 2011, p. 3, disponible à l'adresse suivante : https://perma.cc/RT5Y-B9QK.

110 Voy. Better Finance, Pension Savings: The Real Return, 2019, p. 98, disponible à l'adresse suivante : https://perma.cc/4YYF-HZGA. Les secteurs dans lesquels la couverture seraient la plus faible incluraient les
} 
revenu de pension pour les différentes catégories de salariés couverts. Dans un audit de $2015^{111}$ confirmé en $2016^{112}$, la Cour des Comptes avait déjà souligné que « les réserves sont très inégalement réparties entre les travailleurs et seule une minorité des réserves acquises donnera lieu à un complément significatif à la pension légale ${ }^{113}$. Dans la mesure où la PLCS ne permet vraisemblablement pas de contribuer dans un produit à une hauteur similaire à celle d'un produit du deuxième pilier collectif et qu'elle dépend du niveau de salaire du travailleur, il est probable qu'elle présente ce même trait inégalitaire dénoncé par la Cour des Comptes.

\title{
4. Conclusions
}

45. Le choix du gouvernement d'opter pour la création de la PLCS est avant tout politique. Dans la mesure où les critiques évoquées ci-dessus partent du postulat qu'il est préférable de renforcer le deuxième pilier collectif, elles sont fondées. Il est regrettable que ces critiques émises durant le processus législatif n'aient pas permis d'aboutir à un produit tendant à renforcer clairement - même à long terme - le deuxième pilier collectif. On aurait pu envisager la possibilité pour le Gouvernement d'imposer à un employeur la reprise au titre d'un plan du deuxième pilier collectif, les PLCS constituées par ses salariés une fois qu'un pourcentage déterminé de souscription aurait été atteint parmi ces salariés. On pourrait ainsi envisager la PLCS comme un signal envoyé aux employeurs reflétant l'importance du nombre de salariés désireux de cotiser au sein d'un deuxième pilier. Ce signal pourrait marquer le début de démarches visant à élargir le deuxième pilier collectif au sein de l'entreprise.

46. Les prochaines données disponibles concernant le succès de la PLCS permettront peut-être de fournir un meilleur éclairage sur la réponse des salariés et des fournisseurs de produits au titre de la PLCS et par la suite d'en corriger quelques éléments.

47. De manière plus générale, la création de la PLCS a remis en avant certains défauts du deuxième pilier et notamment les inégalités qui persistent entre travailleurs, qui devraient être traités afin de permettre aux revenus de pension issus du deuxième pilier de réellement jouer un rôle de complément à la pension légale.

\author{
$* * *$

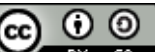 \\ This paper (C) Sabbadini (2019) is licensed under a \\ Creative Commons Attribution-ShareAlike 4.0 International License \\ https://creativecommons.org/licenses/by-sa/4.0/
}

\footnotetext{
salariés de l'Horeca, les intérimaires, ceux des PME et de certains pouvoirs locaux en Wallonie et à Bruxelles : https://perma.cc/W927-K5G3, p. 10.

${ }_{111}$ Cour des Comptes, Cahier de la sécurité sociale, 2015, p. 129 et suiv., disponible à l'adresse suivante : https://perma.cc/3R92-GH73.

${ }^{112}$ Cour des Comptes, fiche de suivi, 2016, p. 153 et suiv., https://perma.cc/BC73-KW5M.

${ }^{113}$ Cour des comptes, 2015, op. cit., p. 129 et p. 139.
} 